\title{
La gestión de megaeventos desde la perspectiva de distintos stakeholders: un análisis exploratorio sobre voluntarios
}

\author{
TERESA FAYOS* \\ MARTINA G. GALLARZA** \\ DAVID SERVERA*** \\ ELENA FLORISTÁN****
}

* Doctora en Ciencias Económicas y Empresariales. Profesora Titular. Universidad de Valencia, Valencia, España. E-mail: teresa.fayos@uv.es. ORCID: 0000-0003-3742-7844. Google Scholar: https://scholar.google.com/ citations?hl=es\&user=ovaOc1oAAAAJ.

** Doctora en Ciencias Económicas y Empresariales. Profesora Titular. Universidad de Valencia, Valencia, España. E-mail: martina.gallarza@uv.es.ORCID:0000-0003-2461-1247.Google Scholar:https://scholar.google.com/citations?hl=es\&user=FyNIRYAAAAJ.


ucv.es. ORCID: 0000-0002-4494-632X. Google Scholar: https://scholar.google.com/citations?hl=es\&user=xzB32roAAAAJ.

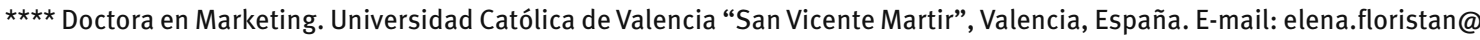
ucv.es. ORCID: 0000-0001-6036-2802. Google Scholar: https://scholar.google.com/citations?hl=es\&user=7ZPE12SAAAAJ. 
COMO CITAR ESTE ARTÍCULO How to cite this article:

Fayos, T. et al. (2019). La gestión de megaeventos desde la perspectiva de distintos stakeholders: un análisis exploratorio sobre voluntarios. Revista Perspectiva Empresarial, 6(1), 41-60

Recibido: 04 de octubre de 2018

Aprobado: 11 de febrero de 2019
RESUMEN Asistimos a un creciente interés académico en el estudio de los eventos. Sin embargo esos estudios se centran principalmente en el análisis de eventos deportivos o culturales y en menor medida en eventos religiosos, a pesar de su potencial de estudio para la gestión y la investigación. Con el presente trabajo buscamos mejorar el conocimiento de los eventos y profundizar en las particularidades de la gestión de voluntarios, stakeholder clave en megaeventos religiosos. Para ello utilizamos una doble metodología cualitativa: entrevistas en profundidad con gestores, combinadas con dinámicas de grupo con voluntarios; todos ellos stakeholders de la XXIV Jornada Mundial de la Juventud celebrada en Madrid. Los resultados obtenidos ilustran la complejidad del proceso de captación, formación y organización de los voluntarios; al tiempo que permiten conocer mejor las motivaciones, intereses y características de los voluntarios de este tipo de eventos.

PALABRAS CLAVE megaevento, evento, stakeholder, voluntarios, cualitativo.

\section{The management of mega-events from the perspective of different stakeholders: An exploratory analysis on volunteers}

ABSTRACT We are witnessing a growing academic interest in the study of events. However, these studies are mainly centered on the analysis of sports or cultural events, and to a lesser extent, on religious events, despite their study potential for management and research. The present work seeks to improve knowledge on events and delve into the particularities of volunteer (stakeholder) management, which is key in religious mega-events. To this end we used a double qualitative methodology: In-depth interviews with managers, combined with group dynamics with volunteers; all of them stakeholders in the XXIV World Youth Days hosted in Madrid. The results obtained illustrate the complexity of the process of volunteer recruiting, training and organization; while at the same time allowing to know better the motivations, interests and characteristics of the volunteers in this type of events.

KEY WORDS Mega-event, event, stakeholder, volunteers, qualitative. 


\section{A gestão de megaeventos desde a perspectiva de distintos stakeholders: uma análise exploratória sobre voluntários}

RESUMO Assistimos a um crescente interesse acadêmico no estudo dos eventos. Porém esses estudos se centram principalmente na análise de eventos esportivos ou culturais e em menor medida em eventos religiosos, a pesar do seu potencial de estudo para a gestão e a investigação. Com o presente trabalho buscamos melhorar o conhecimento dos eventos e aprofundar nas particularidades da gestão de voluntários, stakeholder, chave em megaeventos religiosos. Para isto utilizamos uma dupla metodologia qualitativa: entrevistas em profundidade com gestores, combinadas com dinâmicas de grupo com voluntários; todos eles stakeholders da XXIV Jornada Mundial da Juventude celebrada em Madri. Os resultados obtidos ilustram a complexidade do processo de captação, formação e organização dos voluntários; ao mesmo tempo que permitem conhecer melhor as motivações, interesses e características dos voluntários deste tipo de eventos.

PALAVRAS-CHAVE megaevento, evento, stakeholder, voluntários, qualitativo. 


\section{Introducción}

La utilización de eventos como actos de duración limitada que potencien atributos como el conocimiento, la imagen y la rentabilidad de un destino es un fenómeno creciente, ya que son numerosos los beneficios que conlleva (Lee and Taylor, 2005; Monga, 2006; Gallarza et al., 2010). Sin embargo los estudios realizados hasta la fecha sobre gestión de eventos se han centrado en los eventos deportivos (Farrell, Johnston and Twynam, 1998; Strigas and Newton-Jackson, 2003; Cuskelly et al., 2004) o culturales (Saleh and Wood, 1998; Elstad, 2003); mientras no lo han hecho en los eventos religiosos, a pesar de su potencial tanto de gestión (suelen ser actos muy multitudinarios y por lo tanto lucrativos para la comunidad huésped) como para su análisis académico (la implicación de los asistentes es muy alta). Pues, aproximadamente, 600 millones de viajes religiosos se producen anualmente en el mundo (UNWTO, 2014).

Si bien es cierto que el turismo religioso en su forma más obvia, peregrinaciones, es una de las prácticas turísticas más antiguas (Jackowski and Smith, 1992) que ha generado literatura entre los estudiosos del turismo (Sharpley and Jebson, 2011), en el ámbito del turismo de eventos los eventos religiosos se encuentran en minoría frente a los deportivos o culturales. La delimitación conceptual de lo que es un evento (en magnitud y temporalidad) no es unánime en la literatura, y tampoco lo es su categorización en tipologías, pudiéndose considerar los eventos religiosos como eventos culturales (Ritchie, 1984), a pesar de tener peculiaridades propias (Gallarza et al., 2016). El interés conceptual por el ámbito del evento religioso es, por tanto, notable para el investigador en turismo. A este se añade un interés por el impacto económico que el turismo religioso puede generar (Barajas, Salgado y Sánchez, 2012), dentro del que señalamos un interés adicional del evento religioso como un ámbito específico de gestión.

No obstante, sí es observable en la literatura sobre gestión de eventos destacar la dificultad de coordinar diferentes stakeholders (Shone and Parry, 2004). Esta dificultad se suma a la ya habitualmente señalada necesidad en turismo de combinar diferentes grupos de interés (Lee, Riley and Hampton, 2010), lo que en el caso de los eventos se agrava por el condicionamiento de una fuerte limitación temporal.

Esta realidad del escenario de investigación del evento religioso, conceptualmente difuso pero con fuerte potencial académico y de gestión, es el que nos lleva al planteamiento de este trabajo con un doble objetivo: (i) ampliar el conocimiento sobre la noción de evento ahondando en la conceptualización y tipificación del mismo y (ii) profundizar en las dificultades e idiosincrasia de su gestión desde la perspectiva de los agentes que intervienen; con un notable interés por la particularidad de los eventos religiosos y por la gestión de los voluntarios. Estos últimos son un stakeholder que posee características muy específicas (alta implicación con posible falta de profesionalidad) que los hacen diferentes como fuerza laboral (Gallarza et al., 2009). Para alcanzar dichos objetivos, en la primera parte de este trabajo se revisan los tipos de eventos y sus taxonomías. En la segunda se estudian los eventos desde la perspectiva de la gestión, considerando distintos agentes implicados; para lo que se realiza una investigación cualitativa a través de entrevistas en profundidad con responsables de gestión y dinámicas de grupo con voluntarios para ahondar en el conocimiento de la percepción de estos stakeholders involucrados en un gran evento. Trabajos académicos recientes, reconociendo la dificultad de estudiar comportamientos turísticos novedosos, proponen aproximaciones cualitativas (Park, 2010; Spencer, 2010; Hunter, 2011; Lo and Lee, 2011). Se presentan en el trabajo los resultados de esta fase cualitativa de investigación realizada en torno a un megaevento religioso que tuvo lugar en Madrid, y se recogen las conclusiones a modo de recomendaciones para la gestión en la organización de eventos y futuras líneas de investigación académica.

\section{Marco conceptual}

En la literatura académica de turismo se reconoce que la organización de grandes eventos puede resultar clave para el marketing y desarrollo 
estratégico de los destinos (Lee and Taylor, 2005). La promoción habitual de los destinos, en ocasiones, puede verse notablemente intensificada por la organización de un evento (Monga, 2006). Utilizar eventos de duración limitada que potencien atributos como el conocimiento, la imagen y la rentabilidad de un destino es un fenómeno creciente (Ritchie, 1984; Getz and Wicks, 1994); pues son numerosos los beneficios que conlleva, especialmente en el entorno de competencia entre las ciudades (Muñiz, Cervantes y Abad, 2003). Por ello proponemos revisar la literatura para aproximarnos a dicho concepto desde el ámbito de estudio del marketing de destinos, ya que el evento se circunscribe a un espacio concreto que es el destino. La literatura académica sobre eventos, ya sea próxima al área de la organización de empresas o más a la de marketing turístico, se entronca siempre en la literatura sobre destinos; la cual utilizaremos como marco de referencia para comprender mejor la idiosincrasia de un evento.

\section{Conceptualización y tipología de los eventos}

Conceptualmente lo que podría ser una tarea sencilla (definir qué es un evento), no lo es debido a sus múltiples manifestaciones: su casuística es muy amplia, esto hace difícil una definición genérica que englobe todos los matices y riqueza. Las traducciones de la palabra event tampoco son unívocas en la literatura de turismo en español. Así, para el Diccionario de la lengua española, la definición básica de evento es: "suceso importante y programado, de índole social, académica, artística o deportiva". No obstante, la Enciclopedia de turismo traduce la palabra event como un 'acontecimiento' (Jafari, 2000).

Brown y James (2004) indican que existen tantas definiciones de evento como autores; y a esto hay que añadir las distintas conceptualizaciones ofrecidas en relación a términos como "evento especial", 'megaevento' y "evento Hallmark". Los eventos especiales son: "eventos únicos, infrecuentes y que ocurren fuera de las actividades normales. Para los consumidores suponen una oportunidad de diversión, una experiencia social o cultural que va más allá de su experiencia del día a día" (Getz, 2000, p. 210). Para Goldblatt (1997): "un evento especial es un único momento en el tiempo celebrado con un ritual y que satisface unas necesidades" (p. 2), añadiendo así la precisión de ser "único y ocasional". Allen et al. (2002) describen evento especial como "celebraciones, presentaciones o actuaciones con un ritual específico creadas con motivo de una ocasión especial y con el objetivo de conseguir unos determinados objetivos sociales culturales o corporativos" (p. 11). Lo relevante de todas estas conceptualizaciones es la calidad de 'especial', construida según Jago y Show (1999) sobre 14 características: la unicidad; el simbolismo; la hospitalidad; la tradición; la calidad; la autenticidad; la diversidad de objetivos; el espíritu festivo; la satisfacción de necesidades básicas; la tradición; la flexibilidad; la tangibilidad; el ser organizado en torno a un tema y la accesibilidad.

En segundo lugar, el concepto evento Hallmark es un barbarismo que añade al término de evento especial u ocasional la capacidad para crear imagen y notoriedad: es cierto que "algunos eventos han adquirido tal nivel de recuerdo y reputación que la imagen del evento y de la comunidad han llegado a ser inseparables" (Getz, 2000, p. 210). Hallmark event es traducido en Jafari (2000) como "acontecimiento emblemático", en línea con Graham, Goldblatt y Delby (1995) que lo definen como "eventos que marcan un hito histórico" (p. 69). Para Ritchie (1984) son "grandes eventos, de duración limitada, desarrollados fundamentalmente para reforzar el conocimiento o la notoriedad, el atractivo y el beneficio económico de un destino turístico a corto o a largo plazo" (p. 2). Estos eventos basan su éxito en tres características: su unicidad, su status y su capacidad de crear interés y atraer atención; esto ilustra la dificultad de conceptualización puesto que el autor ha necesitado enumerar características para acotar el concepto. Queda así patente la dificultad de conceptualizar de una manera univoca al concepto Hallmark; aunque está relacionado con calificaciones como emblemático, hito histórico y único.

Getz (2005) utiliza el término Hallmark ligándolo al concepto de "imagen del lugar, marketing de lugares, y marca turística” (p. 16). Según Getz (2008) Hallmark es: 
un evento que posee un significado en términos de tradición, atractivo y cobertura mediática para la comunidad anfitriona, le confiere una ventaja competitiva, y con el tiempo, se convierte en algo tan ligado al destino que son difíciles de separar. (p. 407)

Un ejemplo de esta amplia cobertura mediática es la audiencia de los Juegos Olímpicos de Atenas en 2004 (3900 millones de personas y 35000 horas de audiencia) (Rogge, 2004).

Abordamos, por último, el concepto megaeventos. 'Mega' significa grande en griego, y un millón en el sistema métrico decimal. Los megaeventos son por lo tanto eventos de gran impacto o atracción; "traen consigo extraordinarios niveles de turistas, cobertura mediática, prestigio, o impacto económico para la comunidad anfitriona" (Getz, 2000, p. 210). Marris (1987) señala que los criterios para clasificarlos como mega deberían ser el número de visitantes (más de un millón), su coste (mínimo, medio millón de dólares) o su reputación o prestigio (criterio de más difícil concreción). Una de las definiciones más completas es la de Donald Getz, en una conferencia del Swedish Institute for Regional Research, en 1997:

\footnotetext{
acontecimientos planificados de duración limitada que tienen un impacto extraordinario en el área que los acoge, en uno o más de los siguientes aspectos: número de turistas, gasto de los visitantes, notoriedad conducente a un mayor conocimiento y una mejor imagen, desarrollo de infraestructuras y organizaciones relacionadas que incrementan sustancialmente el atractivo y capacidad del destino. (Fayos-Solá, 1997, p. 242)
}

En definitiva, resulta difícil definir megaevento dado que es un concepto relativo que depende de dos factores: del tamaño de la comunidad que lo organiza y del tipo de impacto buscado. Eventos como los Juegos Olímpicos ofrecen gran impacto, pero otros eventos que atraen menos turistas tienen una gran cobertura mediática y resultan ganadores en términos de popularidad.

Los eventos varían mucho respecto a su tamaño y complejidad: pueden ser más simples o pequeños como una fiesta en un pueblo; mucho más grandes, complejos e internacionales como unos Juegos Olímpicos (Shone and Parry, 2004).
Para entender esta complejidad distintos autores han proporcionado tipologías y clasificaciones en función de su diversidad, su sector y su mercado (Ritchie, 1984; Getz, 1997; Goldblatt, 1997; Watt, 1998; Bowdin, Mcdonnell and O'Toole, 2001; Shone and Parry, 2004; Van Der Wagen, 2006; Maure, 2007).

Ritchie (1984) clasifica a los eventos en siete grupos. Las Ferias mundiales: "son eventos, generalmente periódicos, que se llevan a cabo para el intercambio de bienes y servicios" (Getz, 2000, p. 221); son una de las primeras formas de eventos que históricamente se conocen, que surgieron con el objetivo de focalizar la atención en una ciudad de destino y en la mayor parte de los casos incluyen alguna obra de arquitectura que lo singulariza (por ejemplo, la Torre Eiffel para la Exposición Universal de Paris de 1889) (Maure, 2007). Un segundo grupo es el de los Carnavales y festivales: eventos anuales que construyen su reputación a lo largo de los años. El tercer grupo es el de los Eventos deportivos, principal campo de estudio en el ámbito de los eventos; en particular, los Juegos Olímpicos debido a su importancia mediática y económica (Faulkner et al., 2000; Kasimati, 2003; Revuelta, 2006). Sin embargo hay eventos deportivos que congregan audiencias importantes y que se convierten en escaparates promocionales de las ciudades que los albergan (por ejemplo, la maratón de una ciudad). El problema reside, quizá, en cuándo clasificarlo como evento Hallmark o megaevento. Es claro el caso de los Juegos Olímpicos dado que gracias a su status, su infrecuencia y grandeza entrarían en la categoría de Hallmark; aunque otro tipo de evento deportivo que genere menor afluencia, pero sea igualmente importante, puede resultar difícil de categorizar (Ritchie, 1984).

El cuarto grupo son los Eventos religiosos y culturales, ejecutados sin finalidad comercial pero que suponen una importante contribución al turismo de la región. Ritchie (1984) indica que su principal objetivo quizá no sea fomentar el turismo (por ejemplo, una coronación real), pero si atraen una gran multitud de visitantes. Arcodia y Whitford (2007) recogen la definición de la "South Australian Tourism Commission" (1997): "celebraciones de algo que la comunidad desea compartir y que involucra al público como participantes de la experiencia" (p. 2). Dentro de la literatura, esta es claramente una categoría de menor 
interés de estudio. Sin embargo suelen ser muy multitudinarios y su importancia va en aumento, lo que los justifica como objetivo de este trabajo empírico. Ejemplos de la importancia que han adquirido algunos eventos religiosos son el ḥağğ en La Meca (Arabia Saudí), peregrinación anual musulmana de más de 3 millones de personas; o las visitas del Papa dentro del catolicismo en las que se realizaron misas para más de un millón de personas (Tum, Norton and Wright, 2009; Gallarza et al., 2009, 2010).

Un quinto grupo es el de los Eventos históricos, realizados sobre lugares de reconocido prestigio histórico o patrimonial. El sexto es el de los Eventos comerciales y agrícolas, actividades tradicionales con reconocimiento internacional (por ejemplo, Festival del Tulipán en Holanda). El séptimo es el de los Eventos dedicados a grandes personajes políticos, estos no tienen origen turístico pero la industria local ha ido desarrollando una oferta atractiva alrededor de ellos (por ejemplo, la toma de posesión del presidente de los Estados Unidos).
Sobre la clasificación de Ritchie, Getz (1997) agrupa los eventos en dos grupos: los de dominio público y los de interés privado o individual. Los públicos incluyen a la vez siete tipos: negocios (ferias, exposiciones); lúdicos (juegos, deportes, eventos de recreo); cultural; educativo; arte; político y deportivo. Los de interés privado incluyen dos: las celebraciones personales (aniversarios, vacaciones) y los eventos sociales (fiestas y reuniones). Por último, la clasificación de Maure (2007) aporta más variables de clasificación (tabla 1) y permite describir el evento elegido para el trabajo de campo que acompaña a este trabajo. Así, por su magnitud y características, la Jornada Mundial de la Juventud -JMJ- sería un evento religioso (cultural) según la clasificación de Getz (1997); un evento religioso y cultural según Ritchie (1984); un megaevento según el criterio del tamaño y la importancia del mismo (Getz, 2000) y un evento nacional con participación extranjera, de sede captada, no gubernamental, y del sector cultural, según la clasificación de Maure (2007).

Tabla 1. Matriz de componentes rotados

\begin{tabular}{|c|c|c|c|}
\hline Según su carácter o tipología & $\begin{array}{c}\text { Por su } \\
\text { naturaleza }\end{array}$ & $\begin{array}{l}\text { A partir de su } \\
\text { generación }\end{array}$ & $\begin{array}{l}\text { Según entidades que los } \\
\text { convoquen }\end{array}$ \\
\hline $\begin{array}{l}\text { Congresos, conferencias, simposio, } \\
\text { seminarios, debate, convención, } \\
\text { reunión, panel o debate de experto, } \\
\text { mesa redonda, taller, asamblea, } \\
\text { exposiciones y ferias, festivales, } \\
\text { coloquios, jornadas, eventos } \\
\text { multidestinos, eventos itinerantes, } \\
\text { eventos virtuales, viaje de incentivos }\end{array}$ & $\begin{array}{l}\text { Eventos } \\
\text { internacionales } \\
\text { Eventos } \\
\text { nacionales con } \\
\text { participación } \\
\text { extranjera } \\
\text { Eventos } \\
\text { nacionales }\end{array}$ & $\begin{array}{l}\text { Diseño propio } \\
\text { Sede captada }\end{array}$ & $\begin{array}{c}\text { Gubernamentales } \\
\text { No gubernamentales } \\
\text { Corporativos }\end{array}$ \\
\hline \multicolumn{2}{|c|}{ Según sector generador } & $\begin{array}{l}\text { Según el tamaño o } \\
\text { número de delegados }\end{array}$ & Según sus objetivos \\
\hline \multicolumn{2}{|c|}{$\begin{array}{c}\text { Científico-técnicos } \\
\text { Médicos } \\
\text { ncias sociales y económicos } \\
\text { yricultura y medio ambiente } \\
\text { Culturales } \\
\text { Deportivos y náuticos } \\
\text { Leyes } \\
\text { Educación } \\
\text { Comerciales }\end{array}$} & $\begin{array}{c}\text { Mini eventos (35 a 45) } \\
\text { Pequeños (50 a 249) } \\
\text { Medianos (250 a 499) } \\
\text { Grandes (500 a 2.000) } \\
\text { Mega eventos (> 2.000) }\end{array}$ & $\begin{array}{c}\text { Eventos promocionales } \\
\text { Eventos informativos } \\
\text { Eventos formativos/didácticos } \\
\text { Eventos de refuerzo de } \\
\text { relaciones sociales } \\
\text { Eventos de relaciones internas } \\
\text { Eventos de relaciones } \\
\text { externas }\end{array}$ \\
\hline
\end{tabular}

Fuente: Maure (2007). 


\section{La gestión del megaevento: la importancia del voluntario}

De todos los agentes que intervienen en un megaevento (trabajadores, voluntarios, directivos), el grupo más numeroso es el de los voluntarios (Goeldner, Ritchie and Mcintosh, 2000). Getz (1991) indica que su participación en un evento es crucial para su éxito, aunque son pocos los trabajos que abordan el estudio de su papel como recursos humanos en el ámbito de gestión de eventos. El origen de esta forma de voluntariado podemos encontrarlo en la costumbre de los organizadores de eventos deportivos o culturales en ciudades de implicar a los residentes en la organización como una forma de participación ciudadana (Tosum, 1999, 2006); participación que facilita el desarrollo del turismo, creando mejores oportunidades para la comunidad local, mayores beneficios del desarrollo del turismo y asegurando una mayor satisfacción para locales y turistas (Simmons, 1994).

Existe una creciente dependencia de voluntarios para hacer los eventos viables económica y funcionalmente (Chalip, 2000), lo que presenta a los directores de eventos el reto de su gestión. Cuskelly et al. (2004) señalan que la tendencia de los organizadores de eventos es mirar a los voluntarios como una fuerza de trabajo gratuita y no pensar en mejorar su gestión. De hecho, se tiende a reclutar a más voluntarios de los necesarios sin darse cuenta de las desventajas: incremento de costes (de selección, formación entre otros) y baja satisfacción de los voluntarios al sentirse infrautilizados. Por ello es necesario profesionalizar la gestión de los voluntarios para conseguir mayores niveles de eficiencia y satisfacción tanto de los 'clientes' como de los mismos voluntarios.

A pesar de las dificultades en la profesionalización del sector no lucrativo, Cunningham (1999) señala las presiones que tiene el sector del voluntariado por reevaluar su actual forma de gestión debido a la intensa competencia y escasa financiación; factores que hacen necesario profesionalizar la gestión de estas organizaciones y considerar las estrategias del modelo de recursos humanos del sector privado como un modelo atractivo. En efecto, la tendencia ha sido hacia un creciente reconocimiento de la importancia de aplicar una gestión más profesionalizada por lo menos en lo relativo a la utilización de técnicas comerciales y de marketing (Hankinson, 2001; Dolnicar and Randle, 2004; Wymer, 2003); y se comienza a incorporar dentro de la gestión no lucrativa términos como competencia, posicionamiento o segmentación, no frecuentes hasta ahora. Estos conceptos, no obstante, reciben poca atención por parte de los académicos en la literatura del voluntariado (Dolnicar and Randle 2007; Gallarza et al., 2010).

\section{Peculiaridades y retos en la gestión de los voluntarios en un evento}

Primero se denuncia la falta de conocimientos sobre los factores y procesos que inciden en la permanencia del voluntariado (Dávila y Chacón, 2005), lo que acarrea como consecuencia negativa impedir a las organizaciones rentabilizar los recursos invertidos en los voluntarios. Conocer mejor los mecanismos que lleven a la fidelización de los voluntarios es una cuestión relevante, incluso en el caso de eventos puntuales, por su probable futura participación en otros eventos (Handy et al., 2000) o en su integración como voluntarios habituales en las entidades organizadoras. Segundo el flujo de personas que quieren ser voluntarias no es adecuadamente canalizado, por lo que frecuentemente no se ubican de forma apropiada en una organización ni en una actividad que les permita satisfacer las necesidades que les llevaron a ser voluntarios, renunciando finalmente a seguir intentándolo y abandonando el voluntariado. Todo esto puede llevar no solo a una decepción a nivel personal del voluntario, sino también a una pérdida de recursos por parte de la organización (Dávila y Chacón, 2005). Mejorar las estructuras organizativas del voluntariado se erige como una necesidad en su gestión.

Tercero, nos encontramos con una alta disparidad de voluntarios y organizaciones. Wilson (2000) considera un problema que el término genérico voluntariado abarque un abanico tan amplio y dispar de actividades. Cuarto, las organizaciones que gestionan voluntarios se enfrentan a nuevas tendencias en el mercado del voluntariado como la estabilización y la ralentización de 
la afluencia de voluntarios a las organizaciones y tasas de abandono elevadas (Mota y Vidal, 2003; Vecina, 2001) que tienen consecuencias prácticas en la gestión del voluntariado tanto en reclutamiento como en retención y abandono.

Quinto, también se han señalado dificultades en la gestión de voluntarios diferentes a la gestión de empleados. Vidal y Villa (2007) exponen retos a los que se enfrentan las organizaciones no lucrativas: la creación de un modelo de gestión útil tanto para personas remuneradas como voluntarias; la necesidad de adaptación por parte de las organizaciones ante la aparición de nuevos perfiles de voluntariado; la incorporación de personas provenientes del mundo empresarial y la consolidación de equipos en situación de precariedad económica. Cuestiones con interés creciente puesto que los voluntarios son una pieza clave e insustituible. Getz (1997) apunta que, a pesar de que los voluntarios de eventos son diferentes al resto de los voluntarios, si hay unas características comunes que comparten los voluntarios de cualquier evento que permite conocerlos y gestionarlos mejor: el entusiasmo (los voluntarios normalmente son muy entusiastas con su propio evento); la falta de experiencia y la necesidad de práctica y la búsqueda de diversión y entretenimiento. En este sentido la coordinación de los voluntarios se consolida como una función esencial en la gestión de los mismos. Puesto que ayudan a integrar al voluntario en el propio evento y organizan las funciones diarias de los mismos. El coordinador constituye el contacto directo del voluntario con la organización (Gallarza et al., 2016).

La literatura demuestra la criticidad de la gestión de megaeventos y la importancia del colectivo voluntario en su gestión, así como sus rasgos diferenciales respecto a otros colectivos de trabajadores de un evento, por lo que buscaremos complementar los estudios precedentes analizando de forma empírica algunos de estos aspectos en el caso de gestores y voluntarios (stakeholders) de una JMJ.

\section{Estudio empírico}

La JMJ es un gran evento religioso y cultural que desde 1985, y cada tres años, reúne a jóvenes de todo el mundo durante una semana. Es el evento más internacional y multitudinario que organiza la Iglesia católica en todo el mundo. En la JMJ a analizar asistieron más de un millón de jóvenes procedentes de 139 países de los cinco continentes. Al igual que en otras jornadas aproximadamente un tercio de los jóvenes es del área metropolitana de la ciudad donde se celebra, otro tercio del país organizador y el resto peregrinos de todos los países del mundo; la edad de los jóvenes oscila entre los 15-30 años, con media de 23,9 años, de los cuales $43 \%$ son mujeres y $57 \%$ hombres.

Según los organizadores, participaron en el evento a estudiar 22400 voluntarios; su edad está entre 20 y 23 años; el país que más voluntarios aporta después de España (12185) es Polonia (1048) seguido de Italia (182), México (91) y Francia (81). Los voluntarios se responsabilizaron de funciones de: atención a los participantes y su acogida en estaciones y aeropuertos; información; gestión de plataformas y redes sociales; colaboración en el centro de prensa, traducción e interpretación; logística y acompañamiento de las autoridades y coordinación.

\section{Objetivos y cuestiones a investigar}

Además del objetivo teórico de una mejor comprensión de qué es un evento, el segundo objetivo que planteamos abordar de forma empírica es el de profundizar en las dificultades e idiosincrasia de la gestión de los eventos desde la perspectiva de los agentes que intervienen, particularizando en el caso de los eventos religiosos y la gestión de los voluntarios. Para ello planteamos cuatro cuestiones a investigar con la siguiente justificación.

La gestión de los recursos humanos en eventos, y más concretamente en megaeventos, se caracteriza por su complejidad: manejar diferentes agentes en un tiempo y espacio muy limitados (Tum, Norton and Wright, 2009; Getz, 2005; Getz and Wicks, 1994; Bowdin, Mcdonnell and O'Toole, 2001), por lo que la primera cuestión a investigar 
que planteamos de carácter introductorio y general es:

CI1. ¿Es la gestión de las personas implicadas en la celebración de un megaevento, como la JMJ, una tarea compleja por la diversidad de agentes implicados?

De manera más concreta en lo que respecta a la gestión de estos recursos humanos, el personal de un evento es una parte importante de la calidad del mismo; por ello los gestores del evento tienen que ser conscientes de la importancia de tener al personal satisfecho dado que son ellos los que interactúan con el usuario (Tum, Norton and Wright, 2009), siendo necesario medir sus expectativas y experiencia (Fayos Gardó et al., 2014). Encontramos aquí, en este contacto personal e interacción propios de la experiencia del evento, una particularidad del evento como servicio y una clave de la importancia de todos los agentes implicados en su organización y entre ellos la de los voluntarios que entran en contacto con los asistentes. Además, "el voluntariado es uno de los factores clave en el éxito de un evento. Comprende el trabajo duro y el respaldo de la comunidad para asegurar que un festival o un evento se desarrolla con normalidad" (Goeldner, Ritchie and Mcintosh, 2000 , p. 235). Explorar esta realidad en el caso de la JMJ se apoya en la siguiente cuestión:

CI2. ¿Depende en gran medida el éxito de un evento religioso, como la JMJ, del personal contratado para su celebración y especialmente de los voluntarios?

El siguiente aspecto que deseamos explorar de manera cualitativa, y que da lugar a nuestra tercera cuestión a investigar, es el relativo a la dualidad cognitivo/afectiva que existe en la participación de voluntarios en un evento religioso: si bien pudiera parecer a priori que la mera voluntad es suficiente, tanto para el reclutamiento como para la satisfacción posterior de los voluntarios, las investigaciones anteriores señalan que el voluntario espera poder combinar la motivación de pasarla bien (hedonista) y de relacionarse (social); ambas afectivas con una organización bien llevada en la asignación de tiempos y tareas, elementos estos de carácter cognitivo. Todas estas facetas han sido reseñadas de manera teórica por Getz (1997) o refrendadas en estudios empíricos (Monga, 2006; Gallarza et al., 2009; Cnaan,
Heist and Storti, 2017). Como afirman Gallarza et al. (2010) a la luz de sus resultados sobre la dimensionalidad de la experiencia de voluntario: "las organizaciones públicas y privadas interesadas en la organización de eventos, pueden ver [...] pautas para el reclutamiento y motivación de los voluntarios: la gestión de su tiempo es crítica y su dimensión afectiva no debe ser despreciable" (p. 163). En este trabajo deseamos explorar esta dualidad tanto en la expectativa de los voluntarios como en la consciencia de los organizadores a la hora de reclutar y motivar a los voluntarios. Así, se plantea:

CI3. ¿Buscan los voluntarios una experiencia hedonista y de relación social, pero en la que también son importantes aspectos de organización y asignación de tiempos y tareas?

Una última cuestión, ligada a la dimensión social del voluntariado en eventos, es la predisposición a la recomendación y a la repetición de la acción. La literatura reconoce que el buen ambiente es necesario entre los propios voluntarios, "un ambiente de camaradería entre los propios voluntarios de un evento, puede incluso llevar al mutuo reclutamiento para futuros eventos ya que así participan juntos" (Handy et al., 2000, p. 40); esto además ha resultado confirmado en estudios empíricos sobre voluntariado, con puntuaciones muy elevadas en la medición de variables de lealtad actitudinal y comportamental (Gallarza et al., 2010). Por ello proponemos:

CI4. ¿Se espera que la labor de voluntario en un evento sea tan reconfortante que incentivará la recomendación a otros voluntarios, así como el deseo de repetir la experiencia?

\section{Metodología}

La investigación cualitativa ha sido una herramienta de notable utilidad para el investigador en turismo; en donde la riqueza de la experiencia turística para el individuo (personal, social, emocional, intelectual, cultural) es en ocasiones difícilmente aprehensible en un simple cuestionario. A la corriente que aboga por un retorno de la investigación cualitativa en marketing (Gummesson, 2005) se ha sumado en la literatura turística 
reciente novedosas propuestas metodológicas cualitativas especialmente en investigación de turismo cultural (Hunter, 2011; Park, 2010; Spencer, 2010) frente a un exceso de métodos estadísticos en los que el cómo acaba primando sobre el objetivo (Gummesson, 2000).

Para mejorar la fiabilidad de los resultados, utilizamos una doble metodología cualitativa: entrevistas en profundidad combinadas con dinámicas de grupo, ya que:

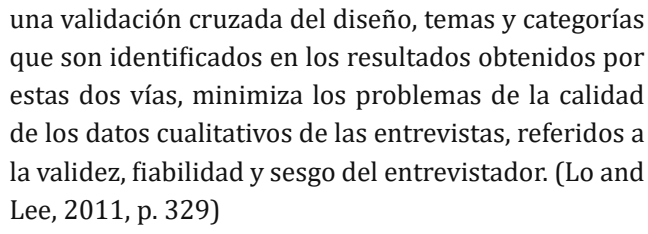
que son identificados en los resultados obtenidos por estas dos vías, minimiza los problemas de la calidad de los datos cualitativos de las entrevistas, referidos a la validez, fiabilidad y sesgo del entrevistador. (Lo and Lee, 2011, p. 329)

Las entrevistas en profundidad y las dinámicas de grupo constituyen métodos similares, aunque para el segundo el interés radica en las interacciones y discusiones entre los miembros del grupo, entretanto la entrevista en profundidad permite tratar temas complejos en esencia (Veal, 2006). Ya que nuestro tema de estudio (gestión de voluntarios en megaeventos) es reconocido como complejo y polifacético, la entrevista en profundidad se erige como método adecuado a combinar con dinámicas de grupo para conocer las dos caras del fenómeno de voluntariado. Las dinámicas, a su vez, aseguran una información variada al recoger y contrastar diferentes opiniones de entrevistados con diferentes backgrounds. Por ello la selección de los participantes en las dinámicas es importante: debe ser heterogénea para fomentar la diversidad de opiniones, pero reagrupando a los participantes en relación a sus inquietudes y comportamientos (Veal, 2006). Así, en nuestro caso, la selección de los participantes se hizo sobre la base de su afiliación como voluntarios: pertenencia a una ONG o a un movimiento católico y participación en anteriores jornadas (dinámica 1) y voluntarios varios de carácter social, universitarios y sin experiencia previa (dinámica 2 y 3); el tipo de muestreo seguido es el llamado exploratorio ad libitum (Soler, 1997), al no existir ningún sistema previo de categorías (solo la condición de voluntario de la JMJ). Los perfiles de ambos colectivos se recogen en las tablas 2 y 3 .

Tabla 2. Perfil de los profesionales (entrevistas en profundidad)

\begin{tabular}{|l|c|l|}
\hline Persona entrevistada & Edad & Cargo \\
\hline Entrevistada A & 29 & $\begin{array}{l}\text { Responsable de comunicación con voluntarios y asistente } \\
\text { del Director General de Voluntariado }\end{array}$ \\
\hline Entrevistado B & 38 & Directora de formación de los voluntarios \\
\hline Entrevistado C & 36 & Director General de Voluntariado \\
\hline
\end{tabular}

Fuente: elaboración propia por parte de los autores. 
Tabla 3. Perfil de los voluntarios (dinámicas de grupo)

Dinámica de Grupo 1

\begin{tabular}{|c||c|l|}
\hline Sexo & Edad & Experiencia en voluntariado \\
\hline Mujer & 17 & Voluntario en ONG y pertenencia a movimiento católico \\
\hline Hombre & 23 & Ha colaborado como voluntario social y en Jornadas anteriores \\
\hline Mujer & 21 & Colaboración como voluntario en Jornadas anteriores \\
\hline Hombre & 22 & Colaboración como voluntario en Jornadas anteriores \\
\hline
\end{tabular}

Dinámica de Grupo 2

\begin{tabular}{|c||c|l|}
\hline Sexo & Edad & Experiencia en voluntariado \\
\hline Mujer & 19 & $\begin{array}{l}\text { Apoyo académico a niños y colaboraciones puntuales en } \\
\text { comedores sociales }\end{array}$ \\
\hline Mujer & 18 & $\begin{array}{l}\text { Apoyo académico a niños. } \\
\text { Colaboración en una residencia de ancianos. }\end{array}$ \\
\hline Mujer & 18 & Colaboración con el voluntariado del colegio \\
\hline Hombre & 25 & Colaboración en una ONG \\
\hline
\end{tabular}

Dinámica de Grupo 3

\begin{tabular}{|c|c|l|}
\hline Sexo & Edad & Experiencia en voluntariado \\
\hline Mujer & 27 & Primera experiencia de voluntariado \\
\hline Hombre & 19 & Ha colaborado como voluntario social \\
\hline Hombre & 18 & Colaboración como voluntario en un convento \\
\hline Hombre & 18 & Colaboración como voluntario social \\
\hline
\end{tabular}

Fuente: elaboración propia por parte de los autores.

La información recogida en las entrevistas y dinámicas fue grabada, transcrita e incorporada en un software CAQDAS para ser trabajada mediante el análisis de contenido, buscando similitudes y particularidades. Análisis adecuado para diseños cualitativos como el nuestro cuando el propósito es el de recoger información sobre factores y manifestaciones de un fenómeno complejo tal como es la organización de eventos (Creswell, 2007). Dicho análisis de contenido se ajusta al enfoque de análisis teórico temático propuesto por Brown y Clarke (2008). De las cuestiones a investigar planteadas, las dos primeras han sido exploradas principalmente en las entrevistas en profundidad puesto que afectan a la gestión de eventos. Es decir se trata de cuestiones vinculadas al desarrollo profesional del voluntario en el evento, siendo en este caso la entrevista la técnica que permite obtener más información. Aunque estas cuestiones también se trataron en las dinámicas. En cuanto a la tercera y cuarta cuestión se ha obtenido información tanto en entrevistas como en dinámicas de grupo (tabla 4).

\section{Resultados y discusión}

Las entrevistas con altos responsables de la organización de los voluntarios duraron entre 50 minutos y una hora, siguiendo un guion predefinido correspondiente a las cuestiones a investigar; aunque se fomentó la participación libre de los entrevistados recogiéndose mucha más información de carácter valioso.

El primer tema tratado en las entrevistas fue la complejidad de la organización del evento y la organización de los voluntarios, recogiéndose ideas comunes relativas a la idiosincrasia de la organización de un megaevento religioso. Reconocer una complejidad en la organización (CI1) fue algo casi espontaneo en todas las entrevistas. "Al principio primaba la estructura diocesana de parroquias, lo que no era muy efectivo: nos costó manejar eso; hemos "retorcido" la organización para que haya un criterio organizativo más lógico" (ENT C).

Dentro de la organización se diferencia claramente entre el perfil de colaborador y el de 
voluntario. El colaborador es la persona que ayuda al evento, pero está menos implicado en su gestión; su implicación se centra más en la tarea a realizar, por ejemplo: las personas que acogen a los peregrinos en sus casas o las organizaciones religiosas que los acogen en sus instalaciones. "La participación del colaborador se centra en tareas secundarias del evento (acogida, información, alojamiento...), no llevan la indumentaria propia del JMJ, se identifican a través de un brazalete, suelen estar vinculados a las parroquias y trabajan de forma bastante autónoma" (ENT A). Los voluntarios son personas que colaboran altruísticamente en la organización y que dependen de ella: están estrechamente vinculados a la organización y esta les asigna las tareas y planifica su trabajo. Los voluntarios se organizan en equipos gestionados por un líder o coordinador cuya función principal es la integración del voluntario en la estructura del evento. Este a la vez reporta a los jefes de área. Estos jefes, formados por personal de la organización, lideran 16 departamentos en los que se divide la organización. Los entrevistados A y C coinciden en la necesidad de mantener los departamentos de manera independiente, aunque interrelacionada, lo que añade dificultad a su gestión.

\footnotetext{
Algunos voluntarios están vinculados a su territorio, y otros están vinculados al evento propiamente dicho: por ejemplo los vinculados a alojamiento, no verán al Papa. Aunque algunos se desapuntan para pasarse a grandes actos, somos estrictos con la permanencia en un grupo. (ENT C)
}

En las entrevistas es recurrente la alusión a la necesaria categorización y jerarquía entre los voluntarios: "si no tuviéramos clara una estructura, esto sería un caos" (ENT A).

Luego se abordó la discusión sobre el trabajo de los voluntarios para conocer cómo se iban a gestionar desde la organización, sus motivaciones y perfil. Respecto a la $\mathrm{CI} 2$, los entrevistados $\mathrm{B}$ y $\mathrm{C}$ coincidieron en que un evento como la JMJ en términos de magnitud se fundamenta principalmente en el trabajo de los voluntarios y los colaboradores; en especial, en tiempos de crisis como hoy. "Desconozco qué se hace en unos JJ.00. pero no puedo imaginar cómo se podría celebrar un acto con más de un millón de peregrinos, sino fuera porque hay una organización de voluntarios detrás" (ENT B).
En la JMJ se reclutaron 22400 voluntarios, principalmente jóvenes españoles y extranjeros, e incluso se rechazaron solicitudes por superar el número necesario. La captación se realizó a través de medios de comunicación, redes sociales y parroquias, siendo muy relevante el boca-oído entre los jóvenes. La selección se realizó a través de entrevistas valorando la actitud positiva hacia los demás y un código mínimo de buena conducta. Los entrevistados matizaron la importancia de transmitir, en las entrevistas de selección, que los voluntarios tenían que tener una actitud de servicio hacia los demás "porque van a trabajar muchas horas en labores que no son las que esperan" (ENT B). Esta motivación permite un mejor desarrollo del trabajo del voluntario porque "limita la posible frustración derivada de realizar trabajos no previstos inicialmente o de no estar cerca del Papa, por ejemplo" (ENT A). Es importante rebajar expectativas para evitar insatisfacción. Esta información resultó coincidente con parte de la literatura anterior sobre voluntariado y eventos (Gallarza et al., 2010).

Una vez seleccionados se realizaron sesiones de formación. En la primera se explicaba la finalidad del evento y el código del voluntario (manual de comportamiento), las áreas de trabajo y las funciones básicas. La formación se consideró muy importante en consonancia con la revisión de la literatura. La voluntad no es condición necesaria y suficiente para el éxito del evento: "son muy buenos, pero también queremos que sean útiles, que sepan operativamente trabajar" (ENT C). Después se hicieron dos sesiones de formación orientadas a los líderes de equipo y una cuarta sobre las tareas concretas a realizar por cada equipo de trabajo. Muy pocos voluntarios habían participado en un evento de tal dimensión, por lo que la organización tuvo que esforzarse para concientizarlos del esfuerzo que supondría su labor concentrada en muy poco tiempo. Se trataba de que sus expectativas se ajustarán a la realidad que encontrarían, "no es lo mismo colaborar en un comedor social dos horas a la semana que realizar jornadas intensivas de 8 horas, el cansancio y el desaliento es mucho mayor" (ENT B). La organización tuvo que hacer también una fuerte labor de concienciación del voluntario, ya que muchos de ellos llegaban con la idea de que "saben mucho y de que van a hacer tareas concretas" (ENT A); "había que hacerles entender que las tareas podían ser muy 
variadas y que era necesario una formación mínima" (ENT B). También se hizo hincapié en que los voluntarios se concienciaran de que eran la imagen del evento y que, por ende, su conducta debía ser ejemplar y seguir las pautas de comportamiento definidas en el código del voluntario. Este es el reverso de la moneda de ese papel clave que el voluntario debe jugar: "su responsabilidad es máxima en todos los sentidos: son la imagen dentro y fuera de la organización" (ENT B).

Las nuevas tecnologías y redes sociales fueron esenciales en la comunicación entre los voluntarios y la organización. Los entrevistados las consideran importantes para reducir la complejidad referida en la CI1, tanto en los procesos de captación como en los de organización y gestión. Las redes sociales contribuyeron decisivamente al efecto 'contagio' entre los voluntarios a la hora de vivir la preparación a la experiencia. Con la discusión en torno a este tema se daba también respuesta a la CI4, relativa a la predisposición a la recomendación y repetición: "según se va acercando el acto, se nos facilita la tarea: mantener la llama viva con tanto tiempo no es fácil, pero entre ellos se animan" (ENT C). La interacción dentro de las redes sociales entre los propios voluntarios y entre estos y la organización ha sido crucial para la motivación, satisfacción e incluso selección.

Por último, en cuanto a las motivaciones y expectativas que han llevado a los voluntarios a proponerse para este evento (CI3), los organizadores consideraban que efectivamente existía una multidimensionalidad (no eran unánimes ni unívocas todas las motivaciones); aunque la dualidad cognitivo/afectiva no ha sido evidente en todos los casos. Si se resaltó principalmente el sentirse útil ayudando a los demás, y el sentimiento de autosatisfacción personal. Además de estas actitudes altruistas también se identificaron elementos hedonistas (diversión, conocer gente) o componentes sociales o cívicos (enseñar la ciudad, acoger a los foráneos): "se insiste para que los peregrinos se sientan cómodos, que conozcan Madrid [...] por eso es Evento Especial [...] se organiza en Madrid pero es para todo el mundo" (ENT C). Si resulta destacable, en la idiosincrasia de la JMJ, su carácter juvenil y por lo tanto la dimensionalidad social: es prioritario el hecho de compartir unos días con gente joven, "es un acto de jóvenes para jóvenes" (ENT A).

Tabla 4. Resultados por Cl y técnica utilizada

\section{Entrevistas en Dinámicas de grupo Profundidad}

\begin{tabular}{llllll}
\hline A & B & C & 1 & 2 & 3 \\
SI & SI & SI & & &
\end{tabular}

Cl1. ¿Es la gestión de las personas implicadas en la celebración de un megaevento, como la JMJ, una tarea compleja por la diversidad de agentes implicados?

\begin{tabular}{|c|c|c|c|c|c|c|}
\hline $\begin{array}{l}\mathrm{Cl} 2 \text {. ¿Depende en gran medida el éxito de un evento } \\
\text { religioso, como la JMJ, del personal contratado para su } \\
\text { celebración y especialmente de los voluntarios? }\end{array}$ & SI & SI & SI & & & \\
\hline $\begin{array}{l}\mathrm{Cl} \text { 3. ¿Buscan los voluntarios una experiencia hedonista y } \\
\text { de relación social, pero en la que también son importantes } \\
\text { aspectos de organización y asignación de tiempos y tareas? }\end{array}$ & $\begin{array}{l}\text { Con } \\
\text { matices }\end{array}$ & SI & $\begin{array}{l}\text { Con } \\
\text { matices }\end{array}$ & SI & $\mathrm{SI}$ & $\mathrm{SI}$ \\
\hline $\begin{array}{l}\text { Cl4. ¿Se espera que la labor de voluntario en un evento sea } \\
\text { tan reconfortante que incentivará la recomendación a otros } \\
\text { voluntarios, así como el deseo de repetir la experiencia? }\end{array}$ & $\mathrm{SI}$ & $\mathrm{SI}$ & SI & SI & $\begin{array}{l}\text { Con } \\
\text { matices }\end{array}$ & $\begin{array}{l}\text { Con } \\
\text { matices }\end{array}$ \\
\hline
\end{tabular}

Fuente: elaboración propia por parte de los autores. 
Las tres dinámicas de grupo estaban formadas por grupos de 4 a 5 voluntarios -jóvenes de 18 a 25 años, implicados socialmente, que han realizado o realizan algún tipo de actividad de voluntariado a nivel local (aunque solo tres de ellos han participado en un evento de tal dimensión)y moderadas por profesores universitarios del equipo de investigación.

Respecto a la CI3 se reconoció que sus motivaciones eran muchas y variadas, aunque no acertaban a 'etiquetarlas' como cognitivas y afectivas. Entre las motivaciones que les han llevado a realizar esas tareas, están en primer lugar las altruistas de ayudar a los demás y poder ser útil: "es bonito ayudar sin que me paguen [...] si buscara dinero, ya no sería igual" (DIN 2). La segunda es la autosatisfacción: "te sientes querido, las sonrisas, te agradecen la compañía, estoy contento de poder ayudar" (DIN 1); resultado coincidente con investigaciones anteriores donde paradójicamente se habla más de 'recibir' que de 'dar' (Gallarza et al., 2009, 2010). También destacan el poder dar ejemplo a los demás, "que el resto de personas se den cuenta de que se puede ayudar" (DIN 3).

Se comenta la variedad de actividades de voluntariado, así como las diferencias entre el voluntariado de larga duración y el previsto en la JMJ que es 'episódico' y por lo tanto diferente. Creen que su participación es diferente porque no es ayudar a necesitados sino apoyar a la organización para que todo se desarrolle correctamente. "Los peregrinos no necesitan tanto apoyo emocional o cariño sino más bien apoyo funcional (orientación, información, consejo, acogimiento)" (DIN 1), por lo que se aprecia una cierta dualidad con predominancia de los aspectos cognitivos.

Respecto a la socialización, el valor social derivado de la experiencia es un factor relevante: "no es tanto el hecho lúdico de conocer a gente nueva sino por la oportunidad de compartir experiencias con gente de la misma edad y que comparte las mismas inquietudes sociales y espirituales que tú" (DIN 3). Es un momento para desarrollar habilidades sociales y compartir puntos de vista distintos en un entorno de diálogo y respeto con personas de diferentes nacionalidades: "no es solo por conocer gente, es porque tienen la misma forma de pensar" (DIN 1). En el caso de los que ya tienen experiencia en eventos internacionales, "ya tienes tema de que hablar [...] estás más a gusto, sabes que piensa como tú" (DIN 1). Se discute sobre esta búsqueda de diversidad, pero a la vez de semejanzas, que se antoja como un eje interesante para profundizar en futuros trabajos; al igual que la posibilidad de dar y recibir en el mismo acto.

La espiritualidad aparece como incentivo relevante, señalan la importancia de que sea un acto religioso y se confiesan practicantes católicos; para ellos es importante que sea un evento católico, así como la presencia del Papa. Es importante relacionarse y compartir unos días con otros creyentes, "sentir que no estás sólo en la fe, que no eres raro" (DIN 3). También es relevante el hecho de ser un ejemplo para el resto de la sociedad (amigos, familiares), no buscan un reconocimiento explícito por parte de estos sino servir de ejemplo: "hombre, no lo haces por eso, pero ellos se alegran" (DIN 2).

Otra motivación es ser buenos anfitriones (orgullo cívico), acoger a los peregrinos, guiarles, enseñarles la ciudad y también el carácter universal del evento: "apetece mucho que sea internacional, nos vamos a tener que comunicar con personas de un montón de países", "ya estoy en Facebook, hablando en inglés con algunos, y es genial" (DIN 1). La motivación social de la internacionalidad se mezcla con la espiritualidad, mostrando la riqueza de la experiencia y la dificultad de delimitar dimensiones estancas: "apetece el tema internacional [...] porque a los españoles los tengo ya muy vistos [...] ver que todos somos cristianos [...] no solo son mis amigas de la parroquia, sino de todas partes" (DIN 2).

En cuanto a la predisposición a la recomendación y a la repetición (CI4) los participantes (DIN 1) se muestran claramente a favor, manifestando que ha sido así en otras ocasiones: "lo aceptas todo, y todo compensa"; o también, en la misma conversación, "no concibo que me pueda defraudar la JMJ, aunque sepa que habrá fallos en la organización" (DIN 1). No obstante, en las dinámicas 2 y 3 , la realidad ha sido distinta; la tabla 4 refleja como esta CI4 ha obtenido resultados contrastados en dinámicas: afirmaciones como "tengo miedo de que no me satisfaga del todo, que me decepcione [...] que no me guste el ambiente" (DIN 1); o también de manera más personalizada como un riesgo personal a correr: "tengo miedo de no estar a la altura de lo que esperan" (DIN 2). 
Sobre la percepción de un coste que limite a la larga su recomendación no son conscientes de que exista un coste en la realización del voluntariado, aunque reconocen que supone tiempo y esfuerzo. Ni siquiera perciben que exista un coste de oportunidad, ya que según afirman es cuestión de organizarse para poder hacer todas las actividades: "cuanto menos tiempo tienes mejor te organizas" (DIN 3).

Respecto a las expectativas sobre la organización de la JMJ, los entrevistados esperaban que se les especificaran con claridad las tareas y que existiera una planificación clara diaria de tareas y responsabilidades. Este es uno de los escasos condicionantes que en las entrevistas 2 y 3 (voluntarios sin experiencia previa en jornadas o grandes eventos religiosos) podrían plantearse a la idea de repetir o recomendar la actividad (CI4). También que no hubiera improvisación por parte de la organización. Miedo a "encontrase solos, un poco perdidos en un evento tan grande" (DIN 3). Esta realidad apunta en la dirección de la complejidad de un evento de estas características y de la necesidad de una planificación previa.

\section{Conclusiones}

De la revisión teórica sobre la conceptualización de los eventos concluimos que resulta complicado concretar el criterio más discriminante que los define y se percibe una falta de delimitación en su tipología dada su enorme riqueza y variedad. De la discusión del estudio empírico con responsables de la gestión y con voluntarios se derivan conclusiones relativas a las particularidades de la idiosincrasia del voluntario de eventos tales como la necesidad de coordinación, la recompensa, la duración y los resultados obtenidos por el voluntario.

La necesidad de coordinación entre los diferentes stakeholders de un evento es una de las primeras conclusiones derivadas de este trabajo. Las inquietudes y prioridades de voluntarios, gestores y visitantes (peregrinos) pueden no ser coincidentes respecto a las expectativas como recientemente señalaban Cnaan, Heist y Storti (2017), pero los gestores y voluntarios apelan a la necesidad de coordinar intereses y no dejar a la libre voluntad el trabajo a realizar. Otros trabajos han planteado la necesidad de profesionalizar la gestión de los voluntarios a fin de conseguir mayores niveles de eficiencia y satisfacción tanto de los 'clientes' como de los voluntarios, afirmación que también se ha derivado de este trabajo. Para facilitar esa coordinación ha resultado muy útil el sistema de coordinadores de equipos de voluntarios y los jefes de área. Sería, por tanto, recomendable que esta estructura se mantenga en nuevos eventos dado que ha demostrado su eficacia en el desarrollo exitoso del evento.

En relación a la recompensa los voluntarios ofrecen sus servicios y tiempo a cambio de asistir al evento, lo que es en cierta manera una forma de remuneración. El desafío para la gestión de eventos es el de utilizar el sistema adecuado de motivación del personal, al igual que ocurre en otro tipo de organizaciones. En línea con Bowdin, Mcdonnell y O'Toole (2001), apuntamos la necesidad de proponer un sistema adecuado de recompensa y reconocimiento que sirva de motivación tanto para el personal como para los voluntarios que trabajan en el evento. Cada evento deberá fijar el sistema de recompensas que mejor se adapte a sus características, siendo las basadas en el reconocimiento las que mejores resultados han dado en nuestro caso.

En la dirección de eventos la corta duración del trabajo de voluntario presenta problemas adicionales derivados, por ejemplo, del hecho de que el personal no haya trabajado de manera conjunta en el evento anteriormente; pues este es una incidencia única que no ha tenido lugar antes, y que por lo tanto no haya suficiente tiempo para crear equipos y socializarse (Shone and Parry, 2004). Esta es una de las dificultades encontradas por los gestores, que sin embargo en el caso del evento religioso de la JMJ parece superarse por la alta implicación de los voluntarios y la categorización de sus tareas. La medición de la percepción real de la experiencia, al término del evento, arrojará mayor luz sobre esta cuestión. De nuevo, la jerarquización de una estructura de gestión con coordinadores de grupo y líderes de área constituye una medida concreta que permite solventar esta dificultad.

En los resultados se muestra que la efectividad de las organizaciones de eventos de voluntarios es alta (Shone and Parry, 2004) debido al gran 
sentido de responsabilidad de los voluntarios, así como a su trabajo y esfuerzo. Los voluntarios van más allá de su trabajo y se implican con la organización, dedicando su tiempo a encontrar recursos o ayudando en la elaboración del evento que participan. La necesidad de medir los impactos de la organización de eventos no solo en términos monetarios sino con otro tipo de medidas se hace necesaria: las llamadas "externalidades positivas no pecuniarias" (Hojman and Hiscock, 2010) son también muy interesantes para todos los stakeholders de un evento: comercios, restaurantes entre otros; y aunque de difícil medición, futuras investigaciones académicas deberían aportar novedosas formas de medición de estos impactos no monetarios tanto en la comunidad de origen en un nivel macro como en cada uno de los stakeholders en un nivel micro.

Son limitaciones de este trabajo las relativas al ámbito (un único evento) y al tipo de muestreo (de conveniencia entre los organizadores del evento y entre voluntarios disponibles). El reducido número de observaciones es también una limitación, aunque se ha buscado aumentar la fiabilidad de los resultados utilizando de forma combinada entrevistas en profundidad y dinámicas de grupo (Lo and Lee, 2011).

Futuras líneas de investigación que pudieran mejorar la aplicabilidad de estos resultados podrían centrarse en proponer comparativas con gestores implicados en la organización de otros colectivos (empleados contratados u peregrinos), lo que arrojaría mayor luz sobre la diversidad de stakeholders de un megaevento.

Creemos que el turismo de eventos es una línea emergente en el horizonte contemporáneo de la investigación turística; auguramos un futuro muy prometedor al turismo religioso puesto que este, al entenderse como altamente experiencial, posee elementos emocionales que necesitan de la combinación de técnicas cualitativas y cuantitativas para su correcta evaluación. En el turismo religioso se dan la mano las experiencias culturales (intra e interculturales), la memoria y la historia. Trabajos recientes ponen en cuestión el valor para determinados turistas de algunas políticas de restauración de memorias históricas y de atractivos turísticos de lugares sagrados (Podoshen and Hunt, 2011), entretanto otros ven posible encontrar experiencias espirituales y religiosas en la práctica de formas profanas tal como el turismo rural (Sharpley and Jebson, 2011). Por ello creemos en el potencial de investigación del acto turístico, si buscamos explorar su dimensión religiosa y espiritual. Tanto de manera explícita en el turismo de eventos religiosos o de peregrinaciones como de manera implícita en algunas prácticas de turismo cultural (ej. visitas a catedrales) e incluso en otras formas de turismo de ocio.

\section{Referencias}

Allen, J. et al. (2002). Festival and special event management. New Jersey, USA: John Wiley \& Sons.

Arcodia, C. and Whitford, M. (2007). Festival Attendance $\&$ the Development of Social Capital. Journal of Convention \& Event Tourism, 8 (2), 1-18.

Barajas, Á., Salgado, J. y Sánchez, P. (2012). Problemática de los estudios de impacto económico de eventos deportivos. Estudios de Economía Aplicada, 30 (2), 441-461.

Bowdin, G., Mcdonnell, A. and O'Toole, W. (2001). Events Management. New York, USA: Routledge.

Brown, S. and James, J. (2004). Event design and management: Ritual sacrifice? En Yeoman, I, Robertson, M. and Ali-Knight, J. (Ed.), Festival and events management: An international arts and culture perspective (pp. 53-64). New York, USA: Routledge.

Brown, V. and Clark, V. (2008). Using thematic analysis in psychology. Qualitative Research in Psychology, 3 (2), 77-101.

Chalip, L. (2000). Sydney 2000 volunteers and the organisation of the Olympic Games: Economic and formative aspects. En Moragas, M., Moreno, A.B. and Puig, N., Volunteers, Global Society and the Olympic Movement (pp. 205-214). Lausanne, Switzerland: International Olympic Committee.

Cnaan, R.A., Heist, H.D. and Storti, M.H. (2017). Episodic volunteering at a religious megaevent: Pope Francis's visit to Philadelphia. Nonprofit Management and Leadership, 28 (1), 85-104.

Creswell, J.W. (2007). Qualitative inquiry and research design. Thousand Oaks, USA: SAGE Publications. 
Cunningham, I. (1999). Changes in the Management of Volunteers Workers: The end of the Well Meaning Amateur? En 5th Researching the Voluntary Sector Conference, National Council for the Voluntary Sector, City University of London, London, England.

Cuskelly, G. et al. (2004). Predicting the behavioral dependability of sport event volunteers. Event Management, 9 (1-2), 73-89.

Dávila, M.C. y Chacón, F. (2005). Diferencias entre voluntarios ambientales y socioasistenciales en el perfil sociodemográfico. Revista de Psicología Social Aplicada, 13 (3), 19-44.

Dolnicar, S. and Randle, M. (2004). What Moves Which Volunteers to Donate Their Time? An Investigation of Psychographic Heterogeneity among Volunteers in Australia. En Conference Proceedings of the Australian and New Zealand Marketing Academy, Wellington, New Zealand.

Elstad, B. (2003). Continuance commitment and reasons to quit: A study of volunteers at a Jazz Festival. Event Management, 8 (2), 99-108.

Farrell, J., Johnston, M. and Twynam, D. (1998). Volunteer motivation, satisfaction, and management at an elite sporting competition. Journal of Sport Management, 12 (4), 288-300.

Faulkner, B. et al. (2000). Monitoring the tourism impacts of the Sydney 2000 Olympics. Event Management, 6 (4), 231-246.

Fayos Gardó, T. et al. (2014). Measuring socio-demographic differences in volunteers with a value-based index: Illustration in a mega event. VOLUNTAS: International Journal of Voluntary and Nonprofit Organizations, 25 (6), 1345-1367.

Fayos-Solá, E. (1997). The Impact of mega events. Annals of Tourism Research, Notes and Reports, 25 (1), 241-257.

Gallarza, M.G. et al. (2009). Consumer behavior in a religious event experience: An empirical assessment of value dimensionality among volunteers. International Journal of Culture, Tourism and Hospitality Research, 3 (2), 165-180.

Gallarza, M.G. et al. (2010). La dimensionalidad de valor en la experiencia de voluntario en un mega-evento turístico. Revista Europea de Dirección y Economía de la Empresa, 19 (4), 149-170.
Gallarza, M.G. et al. (2016). Volunteering for special events: Comparison between expected and perceived values. Innovar, 26 (59), 47-60.

Getz, D. (1991). Festivals, Special Events and Tourism. New York, USA: Van Nostrand Reinhold.

Getz, D. (1997). Event Management and Event Tourism. New York, USA: Cognizant Communication Corporation.

Getz, D. (2000). Festivals and special events: Life cycle and saturation issues. En Garter, W. and Lime, D. (Ed.), Trends in outdoor recreation leisure and tourism (pp. 175-185). Wallingford, United Kingdom: CABI.

Getz, D. (2000). Developing a research agenda for the event management field. En Allen, J. et al. (Ed.), Events beyond 2000: Setting the agenda, proceedings of conference on event evaluation, research and education (pp. 10-21). Sydney, Australia: University of Technology Sydney.

Getz, D. (2005). Event management and event tourism. New York, USA: Cognizant.

Getz, D. (2008). Event tourism: Definition, evolution and research. Progress in Tourism Management, 29 (3), 403-428.

Getz, D. and Wicks, B. (1994). Professionalism and certification for festival and event practitioner: Trends and issues. Festival Management and Event Tourism, 1 (1), 1-3.

Goeldner, R.C., Ritchie, J.R.B. and Mcintosh, W.R. (2000). Tourism. Principles, Practices, Philosophies. New York, USA: Wiley.

Goldblatt, J. (1997). Special events: Best practices in modern event management. New York, USA: Van Nostrand Reinhold.

Graham, S., Goldblatt, J. and Delby, I. (1995). The ultimate guide to Sport Event Management and Marketing. Chicago, USA: Irwin.

Gummesson, E. (2000). Qualitative methods in management research. Thousand Oaks, USA: SAGE Publications.

Gummesson, E. (2005). Marketing de relacionamento total: gerenciamento de marketing, estratégia de relacionamento e abordagens de CRM para a economia de rede. Porto Alegre, Brasil: Bookman. 
Handy, F. et al. (2000). Public perception of "who is a volunteer": An examination of the net-cost approach from a cross-cultural perspective. VOLUNTAS: International Journal of Voluntary and Nonprofit Organizations, 11 (1), 45-65.

Hankinson, P. (2001). Brand Orientation in the Charity Sector: A Framework for Discussion and Research. International Journal of Nonprofit and Voluntary Sector Marketing, 6 (3), 231-242.

Hojman, D.E. and Hiscock, J. (2010). Interpreting suboptimal business outcomes in light of the Coase Theorem: Lessons from Sidmouth International Festival. Tourism Management, 31 (2), 240-249.

Hunter, W.C. (2011). Rukai indigenous tourism: Representations, cultural identity and Q method. Tourism Management, 32 (2), 335-348.

Jackowski, A. and Smith, V. (1992). Polish pilgrim-tourists. Annals of Tourism Research, 19 (1), 92-106.

Jafari, J. (2000). Enciclopedia del turismo. Madrid, España: Editorial Síntesis.

Jago, L.K. and Shaw, R. (1999). Consumer perceptions of special events: A multi -stimulus validation. Journal of travel and Tourism Marketing, 8 (4), 1-24.

Kasimati, E. (2003). Economics aspects and the summer Olympics: A review of related research. The International Journal of Tourism Research, 5 (6), 433-444.

Lee, C. and Taylor, T. (2005). Critical reflections on the economic impact assessment of a mega-event: The case of 2002 FIFA World Cup. Tourism Management, 26, 595-603.

Lee, T.J., Riley, M. and Hampton, M.P. (2010). Conflict and Progress: Tourism Development in Korea. Annals of Tourism Research, 37 (2), 355-376.

Lo, A.S. and Lee, C.Y.S. (2011). Motivations and perceived value of volunteer tourists from Hong Kong. Tourism Management, 32 (2), 326-334.

Marris, T. (1987). The role and impact of mega-events and attractions on regional and national tourism development. Resolutions 37th congress of the AIEST, Calgary. Revue de Turisme, 42 (4), 3-12.

Maure, G. (2007). Definiciones y tendencias del turismo de eventos. Contribuciones a la Economía,
82. Recuperado de http://www.eumed.net/ ce/2007b/gma.htm.

Monga, M. (2006). Measuring motivation to volunteer for special events. Event Management, 10 (1), 47-61.

Mota, R. y Vidal, F. (2003). Solidaridad y morfología de los voluntarios madrileños: informe de investigación. Madrid, España: Comunidad de Madrid.

Muñiz, N., Cervantes, M. y Abad, J. (2003). Análisis de marketing de ciudades a partir de un sistema de indicadores urbanos. Aplicación a la caracterización de ciudades españolas. En XV Encuentro de Profesores Universitarios de Marketing, Córdoba, Argentina.

Park, H.Y. (2010). Heritage Tourism: Emotional Journeys into Nationhood. Annals of Tourism Research, 37 (1), 116-135.

Podoshen, J.S. and Hunt, J.M. (2011). Equity restoration, the Holocaust and tourism of sacred sites. Tourism Management, 32 (6), 1332-1342.

Revuelta, R. (2006). Turismo y eventos deportivos: los Juegos Olímpicos de invierno. Oppidum, 2, 399428.

Ritchie, J.R.B. (1984). Assessing the impact of hallmark events: Conceptual and research segues. Journal of Travel Research, 23 (1), 2-11.

Rogge, J. (2004). Global viewing of Athens 2004 Olympic Games breaks records. En International Sports Television Convention, Monaco, France.

Saleh, F. and Wood, C. (1998). Motives of volunteers in multicultural events: The case of Saskatoon Folkfest. Festival Management and Event Tourism, 5 (1-2), 59-70.

Sharpley, R. and Jepson, D. (2011). Rural tourism: A spiritual experience? Annals of Tourism Research, 38 (1), 52-71

Shone, A. and Parry, B. (2004). Successful event management: A practical handbook. Hampshire, United Kingdom: Cengage Learning EMEA.

Simmons, D.G. (1994). Community participation in Tourism. Tourism Management, 15 (2), 98-108.

Soler, P. (1997). La investigación cualitativa en marketing y publicidad: el grupo de discusión y el análisis de datos. Barcelona, España: Paidós Ibérica. 
South Australian Tourism Commission. (1997). Wine and tourism: A background research report. Adelaide, Australia: South Australian Tourism Commission.

Spencer, D.M. (2010). Case Study. Facilitating public participation in tourism planning on American Indian reservations: A case study involving the Nominal Group Technique. Tourism Management, 31 (5), 684-690.

Strigas, A. and Newton-Jackson, E. (2003). Motivating volunteers to serve and succeed: Design and results of a pilot study that explores demographics and motivational factors in sport volunteerism. International Sports Journal, 7 (1), 111-123.

Tosum, C. (1999). Towards a typology of community participation in the tourism development process. International Journal of Tourism and Hospitality, $10(2), 113-134$.

Tosum, C. (2006). Expected nature of community participation in tourism development. Tourism Management, 27 (3), 493-504.

Tum, J., Norton, P. and Wright, J.W. (2009). Events Management. New York, USA: Routledge.

UNWTO. (2014). Tourism can protect and promote religious heritage. Recuperado de http://media. unwto.org/press-release/2014-12-10/tourism-can-protect-and-promote-religious-heritage.
Van Der Wagen, L. (2006). Human resource management for events: Managing the event workforce. Oxford, England: Butterworth-Heinemann.

Veal, A.J. (2006). Research methods for leisure and tourism: A practical guide. New Jersey, USA: Prentice Hall.

Vecina, M.L. (2001). Factores psicosociales que influyen en la permanencia del voluntariado. Madrid, España: Universidad Complutense de Madrid.

Vidal, P. y Villa, A. (2007). Las personas en las organizaciones del Tercer Sector. Tercera Época, 40, 10-13.

Watt, D. (1998). Event Management in Leisure and Tourism. Harlow, United Kingdom: Pearson Longman.

Wilson, J. (2000). Volunteering. Annual Review of Sociology, 26 (1), 215-240.

Wymer, W. (2003). Differentiating Literacy volunteers: A segmentation analysis of target marketing. International Journal of Nonprofit and Voluntary Sector Marketing, 8 (3), 267-285. 